\title{
The RNA-binding protein hnRNPLL induces a T cell alternative splicing program delineated by differential intron retention in polyadenylated RNA
}

Vicky Cho ${ }^{1,5}$, Yan Mei ${ }^{1}$, Arleen Sanny ${ }^{3}$, Stephanie Chan ${ }^{1}$, Anselm Enders ${ }^{4}$, Edward M Bertram ${ }^{5}$, Andy Tan ${ }^{1,2,3}$, Christopher C Goodnow ${ }^{1,5^{*}}$ and T Daniel Andrews ${ }^{1,5^{*}}$

\begin{abstract}
Background: Retention of a subset of introns in spliced polyadenylated mRNA is emerging as a frequent, unexplained finding from RNA deep sequencing in mammalian cells.

Results: Here we analyze intron retention in T lymphocytes by deep sequencing polyadenylated RNA. We show a developmentally regulated RNA-binding protein, hnRNPLL, induces retention of specific introns by sequencing RNA from T cells with an inactivating Hnrpll mutation and from B lymphocytes that physiologically downregulate Hnrpll during their differentiation. In Ptprc mRNA encoding the tyrosine phosphatase CD45, hnRNPLL induces selective retention of introns flanking exons 4 to 6; these correspond to the cassette exons containing hnRNPLL binding sites that are skipped in cells with normal, but not mutant or low, hnRNPLL. We identify similar patterns of hnRNPLLinduced differential intron retention flanking alternative exons in 14 other genes, representing novel elements of the hnRNPLL-induced splicing program in T cells. Retroviral expression of a normally spliced cDNA for one of these targets, Senp2, partially corrects the survival defect of Hnrpll-mutant T cells. We find that integrating a number of computational methods to detect genes with differentially retained introns provides a strategy to enrich for alternatively spliced exons in mammalian RNA-seq data, when complemented by RNA-seq analysis of purified cells with experimentally perturbed RNA-binding proteins.
\end{abstract}

Conclusions: Our findings demonstrate that intron retention in mRNA is induced by specific RNA-binding proteins and suggest a biological significance for this process in marking exons that are poised for alternative splicing.

\section{Background}

Splicing of introns from pre-messenger RNA is a tightly regulated process performed by the spliceosome [1]. The assembly of the spliceosome at appropriate exon-intron boundaries is influenced by regulatory RNA-binding proteins (RBPs) that operate antagonistically to both enhance and repress this splicing machinery [2-4]. Heterogeneous nuclear ribonucleoproteins (hnRNPs) predominantly

\footnotetext{
* Correspondence: chris.goodnow@anu.edu.au; dan.andrews@anu.edu.au 'Immunogenomics Laboratory, John Curtin School of Medical Research, Australian National University, GPO Box 334, Canberra City ACT 2601, Australia

${ }^{5}$ Australian Phenomics Facility, Hugh Ennor Building, Australian National University, Garran Road, ACT 0200 Canberra, Australia

Full list of author information is available at the end of the article
}

perform a splicing repressor function and their binding blocks spliceosome assembly and leads to exclusion of exons from mature mRNAs. Serine/arginine-rich (SR) proteins bind enhancer sequences in RNA and promote association of spliceosomal proteins at splice sites. This general model nevertheless requires testing in physiological experimental systems where developmentally regulated splicing factors are specifically perturbed, but few such systems have been described, especially for mammalian cells.

Recent information has identified that transcription and splicing are functionally coupled, allowing cross-talk between the two processes [5]. Co-transcriptional assembly of the spliceosome on nascent, chromatin-associated RNA transcripts is a significant factor in correct gene regulation in yeast [6], Drosophila [7] and mammals 
[8-10]. The spliceosome has been shown to quickly associate with chromatin-associated nascent RNA in human HeLa cells [11] and slowing the rate of RNA polymerase II transcription in Drosophila greatly increases the rate of alternative exon inclusion [12]. High-throughput sequencing of chromatin-associated, nascent RNA in Drosophila has revealed that the majority of introns are co-transcriptionally spliced at least half of the time, though a minority of introns are spliced slowly and some appear never to be co-transcriptionally spliced [7]. This variability in co-transcriptional splicing efficiency occurs even within single transcripts and suggests that splicing is regulated at the level of the intron [7], presumably by different RBPs such as the hnRNPs and SR proteins. Introns that are consistently identified to be resistant to co-transcriptional splicing correlate with annotated alternative exons $[7,9,13]$.

To understand mammalian alternative splicing, and define the relationship between variable intron retention after transcription and alternative splicing, it would be useful to be able to experimentally perturb developmentally regulated alternative splicing events through genetic mutations in the specific RBPs that control them. One of the best defined mammalian alternative splicing events occurs in the Ptprc gene encoding the major plasma membrane tyrosine phosphatase, CD45, in T lymphocytes and other blood leukocytes $[4,14]$. In memory $\mathrm{T}$ cells that have been activated previously by antigens, exons 4, 5 and 6 are skipped in the translated Ptprc mRNA. The resulting loss of the CD45$\mathrm{RA}, \mathrm{RB}$ and $\mathrm{RC}$ domains in the extracellular domain of the protein, detected by flow cytometric staining with specific antibodies, is used as the primary marker to differentiate memory $\mathrm{T}$ cells and activated $\mathrm{T}$ cells $\left(\mathrm{CD} 45-\mathrm{RO}^{+}\right)$ from naïve $\mathrm{T}$ cells $\left(\mathrm{CD} 45-\mathrm{RA}^{+}\right.$or $\left.\mathrm{CD} 45-\mathrm{RB}^{+}\right)$. Even in naïve T cell mRNA all three Ptprc cassette exons are rarely included whereas they are all included in B lymphocyte Ptprc mRNA, resulting in the CD45R-ABC isoform (B220) that is detected by specific monoclonal antibodies to identify B cells.

Silencing of Ptprc exons 4, 5 and 6 in T cells requires hnRNPLL, a protein with three RNA-recognition motif (RRM) domains whose mRNA expression correlates with Ptprc exon exclusion: it is highest in $\mathrm{CD}_{4} \mathrm{RO}^{+}$activated and memory $\mathrm{T}$ cells that exclude exons 4 to 6 , at intermediate levels in $\mathrm{CD}_{45} \mathrm{RB}^{+}$naïve $\mathrm{T}$ cells, and at very low levels in CD45RABC ${ }^{+} \mathrm{B}$ cells that include all three exons [15-17]. Mice homozygous for a destabilizing point mutation in the amino-terminal RRM domain, Hrnpll $^{\text {thu }}$, fail to exclude exons 4, 5, and 6 in T-cell Ptprc mRNA and expression of CD45-RA and CD45-RC protein isoforms are increased 50-fold on different T-cell subsets [16]. Likewise, increased inclusion of Ptprc exons 4 to 6 occurs when hnRNPLL is depleted from human T cells by short hairpin RNA (shRNA) expression, while silencing of Ptprc exon 4 is induced in human T cells transfected to overexpress Hnrpll cDNA $[15,17]$. The isolated amino-terminal RRM domain normally binds with sequence specificity and micromolar affinity [16] to an RNA consensus sequence, the activation response sequence (ARS), which mediates exon silencing in activated T cells and occurs in each of Ptprc exons 4, 5 and 6 [18]. Thus, hnRNPLL is a developmentally regulated Ptprc splicing silencer whose expression and activity are critical for the regulated changes in CD45 isoforms on $\mathrm{T}$ and B lymphocytes.

A closely related protein, hnRNPL, has also been shown to bind Ptprc ARS RNA sequences present in exons 4 to $6[19,20]$. T cells from mice homozygous for a knockout of the Hnrpl gene have moderately increased inclusion of exons 4 and 6 , resulting in a four-fold increase in CD45RA expression [21]; compared with a 50fold increase caused by Hnrpll mutation. Thus, hnRNPL and hnRNPLL both contribute to exon silencing but their coordinated actions are only partly understood [4].

The interphase lifespan of Hrnplt thu homozygous T cells is greatly shortened, resulting in decreased numbers of naïve $\mathrm{T}$ cells in the circulation [16]. This effect of hnRNPLL deficiency occurs even in $\mathrm{T}$ cells with a null Ptprc gene [22], indicating that hnRNPLL controls other genes contributing to $T$ cell persistence that have yet to be identified. Here we use this mammalian system to analyze the consequences of perturbing hnRNPLL either by mutation or natural expression differences, as revealed by global mRNA changes measured by RNA-seq. hnRNPLL was required to induce a distinct pattern of intron retention surrounding its known target cassette exons in Ptprc. Differential retention of introns in deeply sequenced RNA provided a signature that could be used to identify other mRNAs requiring hnRNPLL for correct splicing in T cells, and this represents a general, albeit not completely specific, strategy to annotate the transcriptome for exons that are likely to undergo differential splicing.

\section{Results}

\section{Alternative introns are differentially retained in Ptprc transcripts}

In a mouse genetic screen we previously identified hnRNPLL as an essential regulatory factor responsible for skipping of Ptprc exons 3, 4 and 5 in T cells. Thunder mice have a loss-of-function point mutation in the Hnrpll gene that destabilizes the amino-terminal ARS-binding RRM domain of hnRNPLL [16]. To analyze the consequences of Hnrpll ${ }^{\text {thu }}$ upon T-cell mRNA splicing, we performed RNA-seq on mRNA isolated from $\mathrm{CD}^{+} \mathrm{T}$ lymphocytes purified from transgenic OT-1 T-cell receptor (TCR) mice that were either wild type or homozygous for Hrnplt ${ }^{\text {thu }}$. The OT-1 TCR transgenes possessed by this mouse provided a uniform $\mathrm{T}$ cell antigen receptor on the $\mathrm{CD} 8^{+}$ 
$\mathrm{T}$ cells with specificity for a known ovalbumin-derived peptide and ensured that most of the cells were antigenically naïve. This resulted in comparable frequencies of naïve and memory T cells in the Hrnplt ${ }^{\text {thu }}$ animals and the wild-type controls, enabling the analysis to focus on the primary effects of the mutation on splicing and minimize the contribution of Hnrpll-independent differences in mRNA splicing between naïve and memory $\mathrm{T}$ cells.

RNA-seq was performed on biologically independent replicate samples starting with RNA purified from $\mathrm{T}$ cells and using oligo-dT to prime first strand cDNA synthesis. The resulting libraries were each sequenced to a depth of greater than 100 million single-end 125 bp reads on an Illumina GAIIx sequencer, and aligned to the mouse genome (mm9) using TopHat with Bowtie [23]. To confirm that the majority of aligned sequence data came from spliced polyadenylated mRNA, we compared the ratio of intronic reads to exonic reads. For each intron in the 9,162 genes expressed at more than a mean of 20 reads per exonic nucleotide, we calculated the number of reads in the last $25 \mathrm{bp}$ of each intron and divided it by the number of reads in the adjacent first 25 bp of the exon, to yield the ratio of reads across the $3^{\prime}$ splice site (3'SS ratio [7,9]) (Figure 1). This showed that almost all mRNAs are completely spliced with a mode of the intron retention less than 0.01 and a median of 0.019 .

The Ptprc gene contains 33 exons spanning $112 \mathrm{~kb}$ on chromosome 1 (Figure 2a), and is highly expressed in $\mathrm{T}$ cells with a median of $>3,300$ reads per exonic nucleotide in each of our CD8 T-cell RNA-seq datasets. The majority of Ptprc introns were spliced out of $99 \%$ of the sequenced RNA, as illustrated by the large difference in read coverage over constitutive exons 9 to 29 compared to the intervening introns (Figure 2b) and by a median 3'SS ratio of 0.01 for Ptprc introns as a whole. In wildtype $\mathrm{CD}^{+}$cells $\left(\mathrm{CD} 45-\mathrm{RB}^{\text {high }}, \mathrm{RA}^{\text {low }}\right.$ and $\left.\mathrm{RB}^{\text {low }}\right)$ there was strong silencing of exons 4 and 6 , which contain multiple ARS, whereas the more abundant exon 5, encoding the CD45-RB isoform, was better represented in sequence reads. The hnRNPLL-dependence of this differential splicing is readily apparent from Figure $2 \mathrm{c}$ - the two wild-type $T$ cell samples had a mean of 777 and 1,639 reads per nucleotide in exons 4 and 6, respectively, compared with a mean of 3,735 for all Ptprc exons, whereas in the Hnrplt ${ }^{\text {thu }}$ T cells exon 4 and 6 were covered by a mean of 2,747 and 3,766 , respectively, compared to a mean of 3,766 for all Ptprc exons.

Surprisingly, an abundance of reads was also observed in wild-type T cells selectively over Ptprc introns 3, 4, 5, 6 and 7 compared with the other introns in the gene. The median read depths over introns 3, 4, 5, 6 and 7 were 310, 366, 746, 759 and 552 reads, respectively, and were significantly higher $\left(P=1.6 \times 10^{-4}\right.$, Mann-Whitney, $\mathrm{W}=200)$ than the mean depths over all other introns (median read depth of 16). Mutant Hnrpll ${ }^{\text {thu }}$ cells also showed significantly increased reads in the same introns, though at a universally lower level than wild-type cells. Hence, wild-type $\mathrm{CD}^{+} \mathrm{T}$ cells display a greater depth of intronic reads and inversely fewer exonic reads relative to mutant cells through the region of the Ptprc transcript known to undergo alternative splicing. The median read depth over these retained introns in wild-type cells is between 8 to $20 \%$ as abundant as sequence reads over all Ptprc exons. This indicates that, in wild-type $\mathrm{CD}^{+} \mathrm{T}$ cells, approximately 8 to $20 \%$ of the sequenced Ptprc mRNA had not yet removed either exon 4 and 


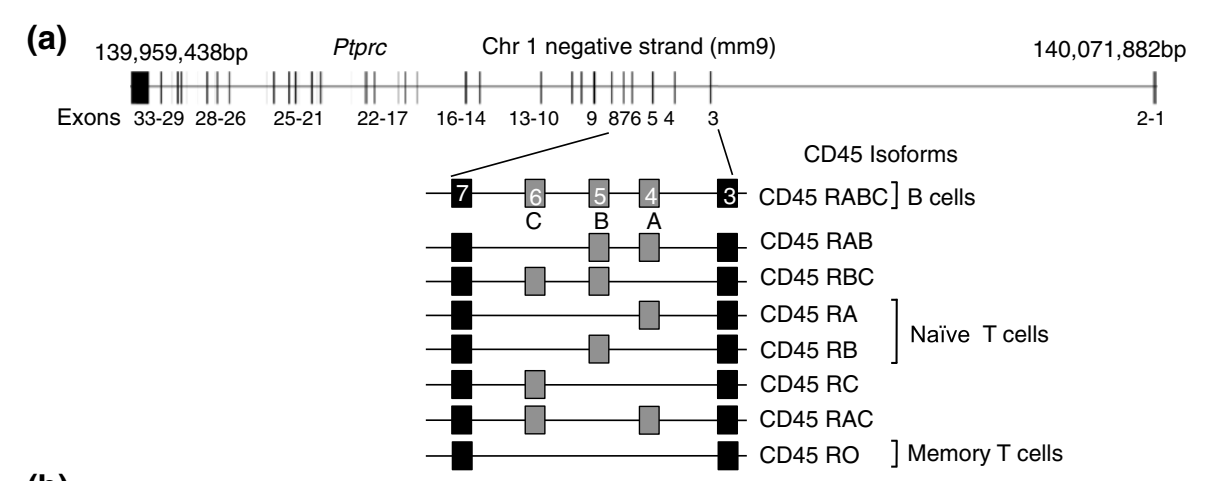

(b)

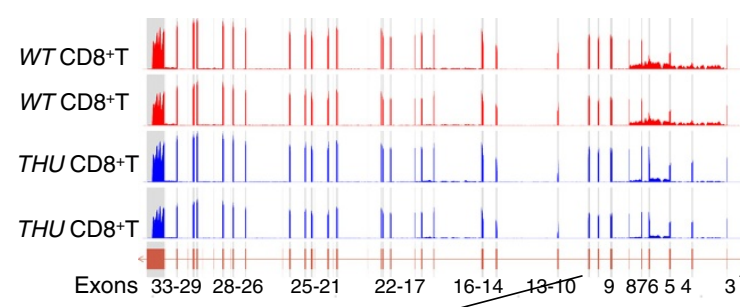

(c)
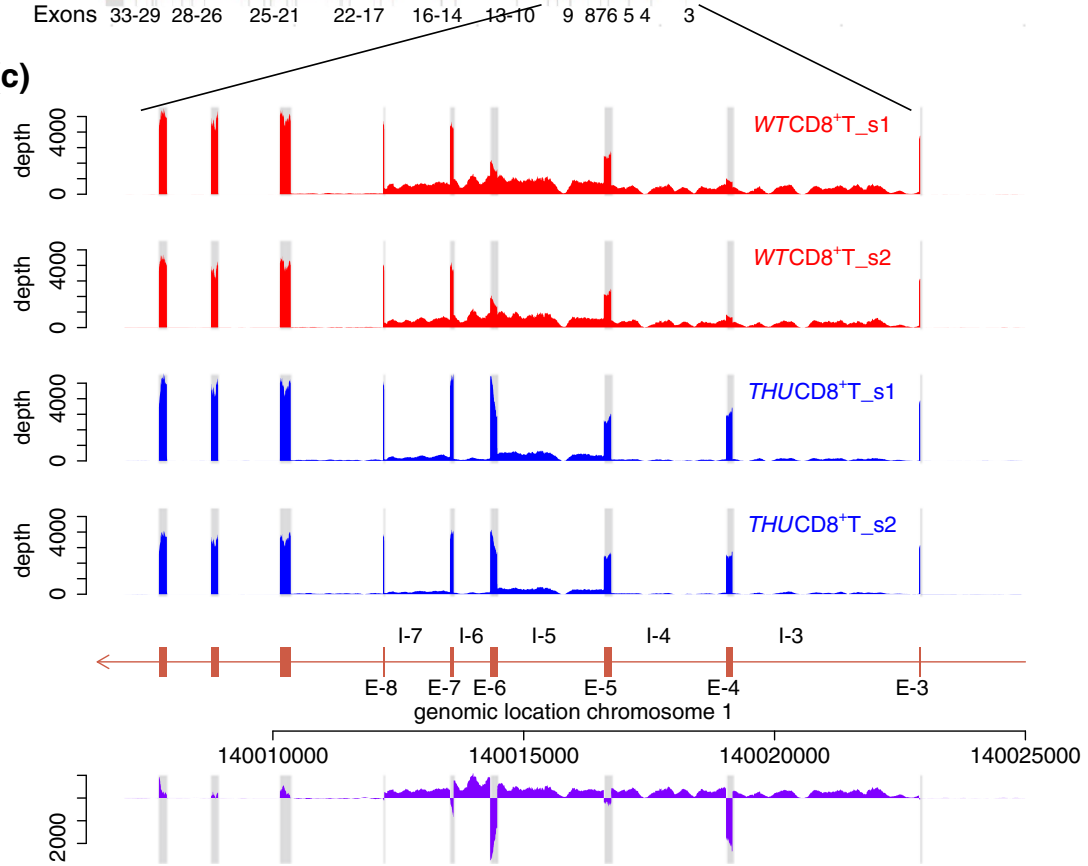

Figure 2 Increased intron retention in wild-type T cells correlates with exon-skipping events in Ptprc. (a) Exons 4, 5 and 6 of the Ptprc (CD45) gene are alternatively spliced in T cells and may be combined to produce eight distinct CD45 isoforms. The longest isoform, CD45RABC, is primarily expressed in B cells whereas T cells express different CD45 isoforms through their development and activation. (b) RNA-seq data along the length of the Ptprc gene show the introns flanking alternative exons 4, 5 and 6 are covered by a greater depth of sequence reads and indicates incomplete splicing of these introns in both wild-type (WT; in red) and thunder (THU; in blue) CD8 ${ }^{+} \mathrm{T}$ cells. (c) Read depth over retained introns is consistent within biological replicate samples, but is different between wild-type (red) and thunder (blue) samples, as shown in the purple trace, which plots the wild-type minus thunder per nucleotide read depth through the alternatively spliced region of the Ptprc gene. Introns and exons are labeled as I- and E-, respectively, followed by their corresponding number. Thunder mutants with a hypomorphic Hnrp/l gene show fewer reads aligned to introns 3, 4, 5, 6 and 7.

exon 6 nor the introns that precede and follow these exons, yet $99 \%$ of the sequenced Ptprc mRNA had removed constitutively spliced introns. It is important to note that deep sequencing of RNA provides a unique opportunity to measure mammalian intron retention in a relatively unbiased manner, in contrast to PCR-based methods that are biased against detecting the very large intronic inserts.

As an independent test of the effect of diminished hnRNPLL activity on lymphocyte mRNA splicing and 
intron retention, we compared RNA-seq data from magnetic-bead enriched $\mathrm{CD} 4^{+} \mathrm{T}$ cells (Hnrpll-high) and CD19 ${ }^{+}$B-cells (Hnrpll-low) (Figure 3a). As expected for bead-based enrichment methods, the B-cell-enriched samples still contained an estimated 7\% $\mathrm{T}$ cells based on mean expression of a set of T-cell-specific mRNAs, assuming exclusive T-cell expression of these genes. Conversely, using the same measure, the T-cell-enriched samples retained an estimated $1 \%$ B cells. In $\mathrm{CD} 19^{+} \mathrm{B}$ cells, the median sequence read depth over Ptprc exons 4, 5 and 6 was 344, which is greater than the median read depth of 165 over all Ptprc exons, demonstrating

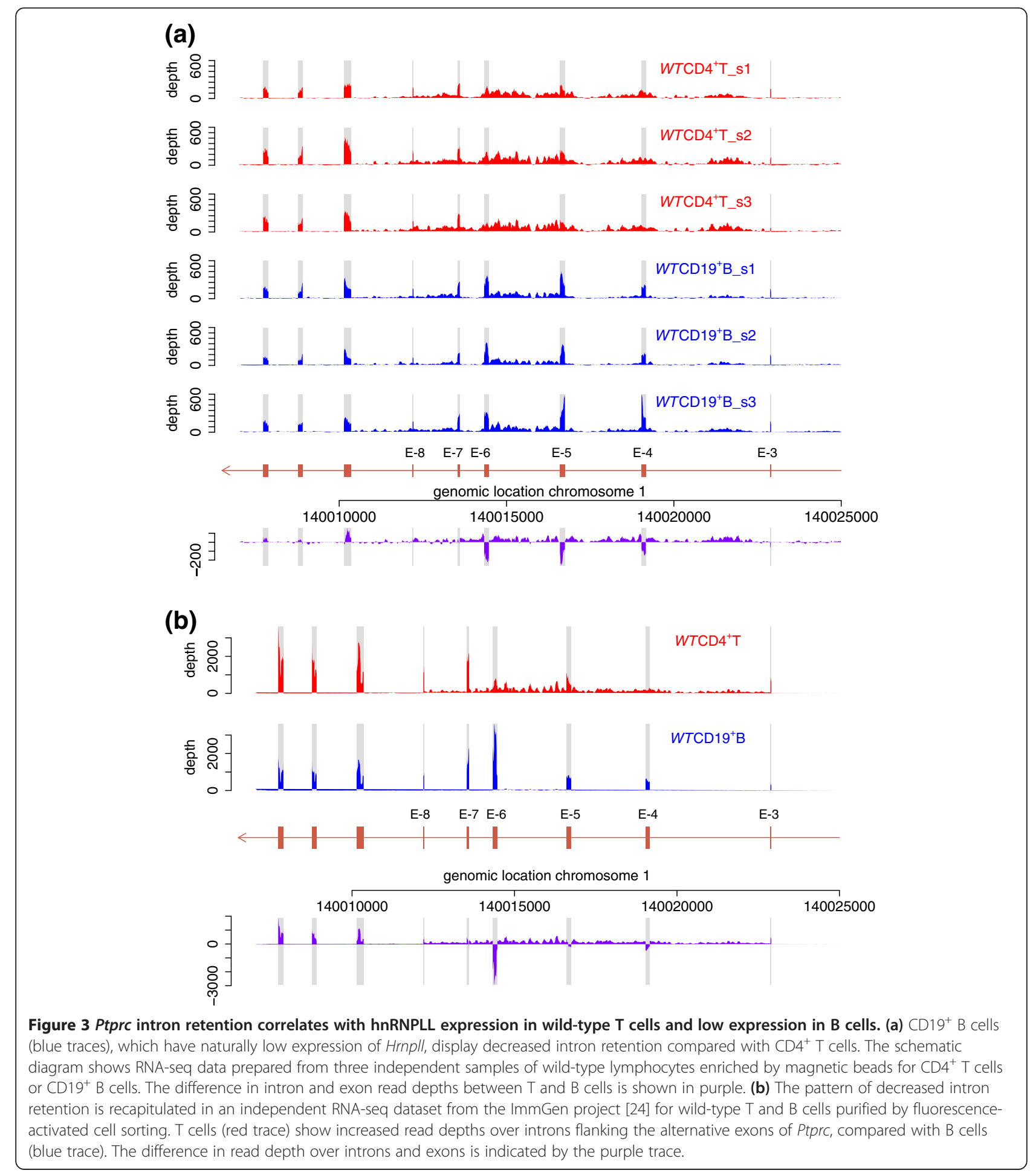


strong inclusion of these three exons as expected, since $\mathrm{B}$ cells express very little hnRNPLL. In $\mathrm{CD} 4^{+} \mathrm{T}$ cells, which do express hnRNPLL, the read depth over exons 4 and 6 was lower (median 130) than the median depth over Ptprc exons as a whole (192), confirming silencing of these exons. A reciprocal pattern was observed for the introns flanking exons 4 to 6 , where there was a higher depth of reads in T cells (median of 54 ) and a lower depth of reads in B cells (median of 23). Hence, B cells with naturally low Hnrpll expression splice out introns 4 to 6 more efficiently than $\mathrm{T}$ cells that express Hnrpll.

As further evidence of differential intron retention between $\mathrm{T}$ and $\mathrm{B}$ cells, we analyzed RNA-seq data generated by the ImmGen project [24] from polyadenylated mRNA isolated from highly purified $\mathrm{CD}^{+} \mathrm{T}$ cells and $\mathrm{CD} 19^{+} \mathrm{B}$ cells prepared using fluorescence-activated cell sorting (Figure 3b) [25]. Ptprc introns 4 to 6 exhibited numerous reads in T cells, with a median of 144 compared to 1,655 for all Ptprc exons. By contrast, very few intron 4 to 6 reads were obtained from B-cell mRNA, with a read depth of 5 compared to a median of 1,071 for all Ptprc exons in the same sample. This indicates that introns 4 through 6 are efficiently spliced out of 99.5\% of Ptprc mRNA in B cells that have little hnRNPLL, but are retained in $9 \%$ of the corresponding mRNA from CD4 T cells.

\section{Retained introns are frequent and correlate with alternatively spliced exons}

Given the findings above, we asked how many other mRNA species show a retained intron in normal mouse $\mathrm{T}$ cells. The 3'SS ratio data shown in Figure 1 suggest that up to $13 \%$ of all introns in polyadenylated mRNA remain unspliced in more than $10 \%$ of the corresponding mRNAs ( $\left.3^{\prime} \mathrm{SS} \geq 0.1\right)$. A smaller subset of introns ( $0.94 \%$ of all introns) remain unspliced in more than $50 \%$ of the mRNAs that contain them. We therefore asked if exons observed with a retained flanking intron commonly undergo alternative splicing, by generating a list of exons that had a 3'SS ratio $\geq 0.1$ in wild-type CD8 $\mathrm{T}$ cells from OT1 mice. In total, 6,797 exons had a 3'SS ratio greater than 0.1, derived from 3,248 genes (Figure 4, Table 1). The median intron retention (IR) ratio in detectable genes was 0.019 in wild-type $\mathrm{T}$ cells. When compared with annotated alternative exons in the Ensembl mouse gene set [26] (NCBIM37, Ensembl release 67), this list showed a highly significant, nonrandom overlap with the alternative splicing categories of cassette exons, mutually exclusive exons and intron retention (according to the nomenclature of [27]) whereas constitutive exons were found significantly less than expected (permutation $P$-value $=0.001$; Figure 4, Table 1). The alternatively spliced Ptprc exons 4 and 6 that are skipped in Hnrpll wild-type T cells had a 3'SS ratio of 0.7 and 0.5 , respectively, placing them within the top $8 \%$ of all exons ranked by largest 3'SS ratio. Hence, 3 'SS ratios as a simple measure of intron retention can be used to enrich for mammalian exons that undergo physiological alternative splicing.

\section{Detection of differentially retained introns in Hnrplt ${ }^{\text {thu }}$ mutants}

The data presented above imply that binding of hnRNPLL to exons in pre-mRNAs delays the excision of flanking introns from polyadenylated mRNA. We therefore sought other hnRNPLL-regulated genes using algorithms to detect differential intron retention or exon exclusion between wild-type and $\mathrm{Hnrpll}^{\text {thu }} \mathrm{CD}^{+}$T cells. Using the DEXSeq method [28] we compared the relative intron read counts within each annotated mouse gene between wild-type and Hnrpll ${ }^{\text {thu }} \mathrm{CD}^{+} \mathrm{T}$ cells from OT1 mice. This identified 138 introns from 114 genes with significantly different numbers of reads between wild type and mutant, including intron 6 from the Ptprc gene, which ranked second most differentially retained according to P-value (Ptprc introns 3, 4, 5 and 7 were also within the top six most significantly retained introns; Additional file 1). Intron 1 of the Ctse gene was more highly retained than all Ptprc introns, and introns from the Ddb2, Senp2, Trim30a and Atp2c1 genes comprised the remainder of the top 10 highest ranked introns by DEXSeq. Visual inspection of reads for these and other highly ranked genes using the Integrative Genomics Viewer (IGV) [29] showed clear cases of differential intron retention between wild-type and Hnrpll $^{\text {thu }} \mathrm{T}$ cells, although these were generally less striking than that observed in Ptprc, most likely due to these mRNAs being less abundant.

To identify differential exon exclusion we used DEXSeq to compare the relative read depths for each exon within annotated mouse genes between wild-type and Hnrplt $^{\text {thu }} \mathrm{T}$ cells, and ranked exons with significant differences according to P-value (Additional file 2). Ptprc exons 4 and 6 were ranked first and second, respectively. Of the 138 introns with DEXSeq evidence of differential intron retention above, 18 of these had at least one flanking exon with evidence of differential exon exclusion. These intron-exon pairs were from the Ptprc, Degs1, Ms4a6c, Cdc42, Il7r, Ctse, Gimap8, Slfn1, Rpl29, Mrpl3 and $I g h g 2 c$ genes.

As a third method to identify hnRNPLL-regulated splicing events, we surveyed differential splice junction usage. We used DEXSeq to compare the reads aligned to all splice junctions between wild-type and Hnrplthu $\mathrm{T}$ cells, which found 588 significant junctions out of 230,416 in total (at a false discovery rate of 0.1 ), threequarters of which show increased usage in $\mathrm{T}$ cells from 
thunder mice, indicating greater splicing diversity is associated with mutant hnRNPLL (Additional file 3). Among 57 junctions that were equally highest-ranked with an adjusted $P$-value of 0 , splice junctions that connect alternative exons in the Ptprc gene were found with a reciprocal pattern of exon-joining versus exonskipping. Junctions joining exon 8 and exon 3, exon 7 and exon 3, exon 7 and exon 5, and exon 5 and exon 3 had higher relative sequence coverage in wild-type $\mathrm{T}$ cells, whereas junctions joining exon 6 and exon 5, exon 5 and exon 4, and exon 4 and exon 3 had higher coverage in Hnrpll $l^{\text {thu }} \mathrm{T}$ cells. Of the top ten genes ranked by evidence of intron retention above, five of these also contained significant differentially altered splice junction usage flanking these introns (Ptprc, Ctse, Trim30a, Rab3gap2 and Slc12a7).

Figure $\mathrm{S} 1$ in Additional file 4 shows a Venn diagram of the overlap of gene identifiers with significant differential

Table 1 Intron retention ratio is correlated with cassette exon, mutually exclusive exon and intron retention splicing events

\begin{tabular}{|c|c|c|c|c|}
\hline $\begin{array}{l}\text { Alternative splicing } \\
\text { event type }\end{array}$ & $\begin{array}{l}\text { Number of annotated } \\
\text { events in ENSEMBL for all } \\
\text { exons }(n=38,911)\end{array}$ & $\begin{array}{l}\text { Number of matched events } \\
\text { for exons with } 3 \text { 'SS IR } \geq 0.10 \\
\qquad(n=6,658)\end{array}$ & $\begin{array}{c}\text { Mean number of matched events } \\
\text { in background }(1,000 \times \text { re-sampling } \\
6,658 \text { exons from } 38,911)\end{array}$ & $\begin{array}{c}\text { Permutation } \\
P \text {-value }\end{array}$ \\
\hline CNE & 19,513 & 2,538 & 3,338 & 0.001 \\
\hline CE & 8,641 & 1,936 & 1,479 & 0.001 \\
\hline MXE & 1,265 & 294 & 216 & 0.001 \\
\hline IR & 3,614 & 749 & 620 & 0.001 \\
\hline A3SS & 1,249 & 208 & 213 & 0.636 \\
\hline A5SS & 1,242 & 243 & 213 & 0.009 \\
\hline
\end{tabular}

Number of alternative splicing event types mapped within 1) all exons in expressed genes and 2) exons having at least $10 \%$ of intron retention in their 3 'SS, and the mean number of the event types within randomly sampled exon sets of the same size. CNE, constitutive exon; $C E$, cassette exon; MXE, mutually exclusive exon; IR, intron retention; A3SS, alternative 3' splice site; A5SS, alternative 5' splice site. 
intron retention, exon inclusion or alternative splice junction ratios. While a total of 9 genes (Ptprc, Ctse, Ighg2c, Wdr82, Ms4a6c, Gnai2, Pik3r4, Degs1, Rrp9) have significant differential intron retention, exon inclusion and altered splice junction usage (and another 51 with just two of these features), they are a minority compared with the total count of genes with one of these features. Hence, either methodology issues and noise in the RNA-seq data may hamper the identification of alternative splicing events associated with intron retention, or intron retention may not always be associated with alternative splicing. It seems plausible that intron retention induced by hnRNPLL binding may delay splicing of certain introns, but may not ultimately alter the splicing fate of the premRNA molecule.

\section{hnRNPLL-dependent splicing in mouse T cells}

The preceding analysis was validated in the sense that it identified known hnRNPLL-dependent splicing events in Ptprc, so we used reverse transcriptase polymerase chain reaction (RT-PCR) to validate highly ranked splicing events in other genes. Ten candidate alternative exons from distinct genes were selected, choosing those genes that ranked highly in one or more of the tests described in the previous section, and showed a clear differential pattern of read distribution between cell types when inspected visually using the IGV [29]. Oligonucleotides complementary to flanking exons were used to amplify PCR products from oligo-dT-primed cDNA prepared from Hnrpll ${ }^{w t}$ and Hnrplt ${ }^{t h u}$ mRNA, and the products analyzed by gel electrophoresis (Figure 5). In addition to Ptprc as a positive control, six of the ten candidate genes, Senp2, Ctse, Trpv2, Ash1l, Slc12a7, Lck, displayed differential band sizes or multiple bands that differed between $\mathrm{T}$ cells with normal or mutant hnRNPLL (Figure 5). Interestingly, these genes all showed evidence for differential exon usage, but none showed bands attributable to retention of a long intron. This likely can be attributed to PCR bias towards shorter products and long intron-containing transcripts not being amplified, which is a bias not shared with RNA-seq. Alternative splicing events found in only three of these genes (Ptprc, Trpv2, Slc12a7) are currently identified by mouse expressed sequence tags (ESTs) from GenBank [30], indicating the need for more comprehensive annotation of alternative splicing by RNA-seq studies.

Browsing the RNA-seq reads data for these genes with IGV indicated that, like Ptprc, three genes (Senp2, Ctse, Slc12a7) appeared to have greater intron retention in wild-type cells than in Hnrplt ${ }^{\text {thu }} \mathrm{T}$ cells, whilst the converse was true for two other genes (Trpv2, Ash1l) and indeterminate for one (Lck) (Figure S2 in Additional file 4). High intron retention and exon skipping occurred in wild-type T cells for Senp2 and Ctse; for Ash1l and
Slc12a7, however, high intron retention in Hnrpll ${ }^{w t} \mathrm{~T}$ cells was associated with exon inclusion. This indicates that either hnRNPLL binding can have both a splicing repressor and enhancer role in wild-type cells, or that potentially some of these genes are indirectly regulated by hnRNPLL or are regulated in concert with another RBP. For all validated candidate genes, the observed alternative splice junction usage from RNA-seq reads supports the alternative products observed with PCR.

For three candidate hnRNPLL targets (Senp2, Ctse and Ash1l) the alternative junctions detected by RNA-seq were outside the significance threshold applied in the DEXSeq analysis (Figure S2 in Additional file 4). Notable from the RNA-seq data for these genes was the enrichment of cryptic junction reads. Generally, cryptic splice sites are rare in the RNA-seq data, but four genes (Senp2, Ctse, Trpv2, Slc12a7) had a diverse number of cryptic splice junctions in their retained introns and flanking the identified alternative exons. Another feature of these differentially spliced genes was minor unannotated alternative exons. Senp2, Ash1l and perhaps Slc12a7 and Trpv2 have differentially included alternative exons that lie within retained introns. Within Senp2, a differentially included, unannotated, alternative exon appears in Hnrplt ${ }^{\text {thu }}$ cells, which is only present at trace levels in wild type. Further investigation of this cryptic exon in Senp 2 by sequencing PCR bands showed that it was included in variable lengths with alternative 3' splice sites (Figure S3 in Additional file 4). Inclusion of this cryptic exon introduced an in-frame stop codon that we estimated caused $23 \%$ of the protein to be truncated in Hnrpll ${ }^{\text {thu }}$ cells (calculated as the percentage of cryptic junctions compared with canonical junctions).

By combining each of the features of hnRNPLLdependent alternative splicing events observed above, we developed a simple scoring scheme based on the sum of Boolean values for each feature (Table 2). These features were intron retention, exon exclusion and splicejunction usage (determined by DEXSeq) along with the presence of cryptic junctions and high 3'- or 5'SS ratios. We scored all introns by these features and ranked them accordingly. Ptprc and four other PCR-validated genes (Senp2, Ctse, Ash1l, Slc12a7) ranked within the top 25, while Trpv2 and Lck ranked 63 and 164, respectively. A further 136 genes had introns with scores that ranked them among the validated genes and were likely candidates for hnRNPLL-dependent splicing. We selected 15 of these candidate genes after visual inspection of the RNA-seq read data with IGV and conducted further RTPCR validation of alternative splicing. The results identified 8 of these 15 genes (Degs1, Sidt1, Mapkapk3, Herc3, Ikbke, Cep110, Mllt6, Rap1gds1) with differential band sizes between hnrpll ${ }^{\mathrm{wt}}$ and $h n r p l l^{\text {thu }} \mathrm{T}$ cells (Figure 6). Table 2 shows the scoring matrix of Boolean values of 
(a) Ptprc

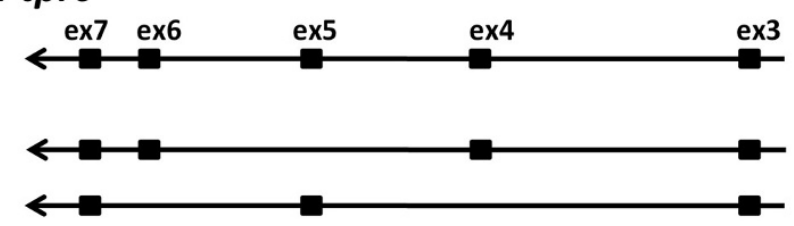

(b) Senp2

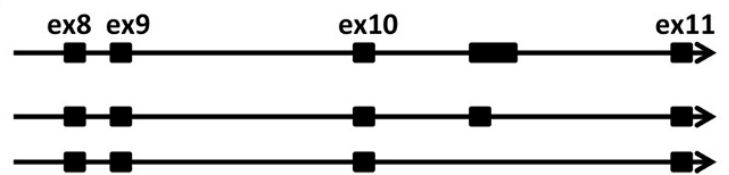

(c) Ctse

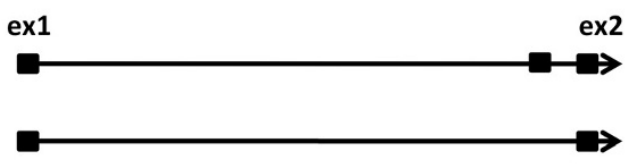

(d) Trpv2

(e) Ash1I
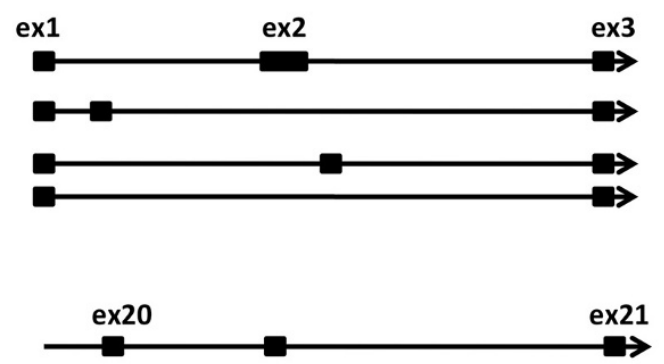

(f) Slc12a7
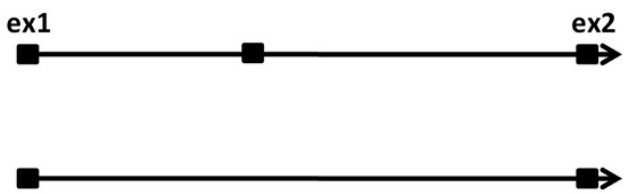

(g) $L c k$
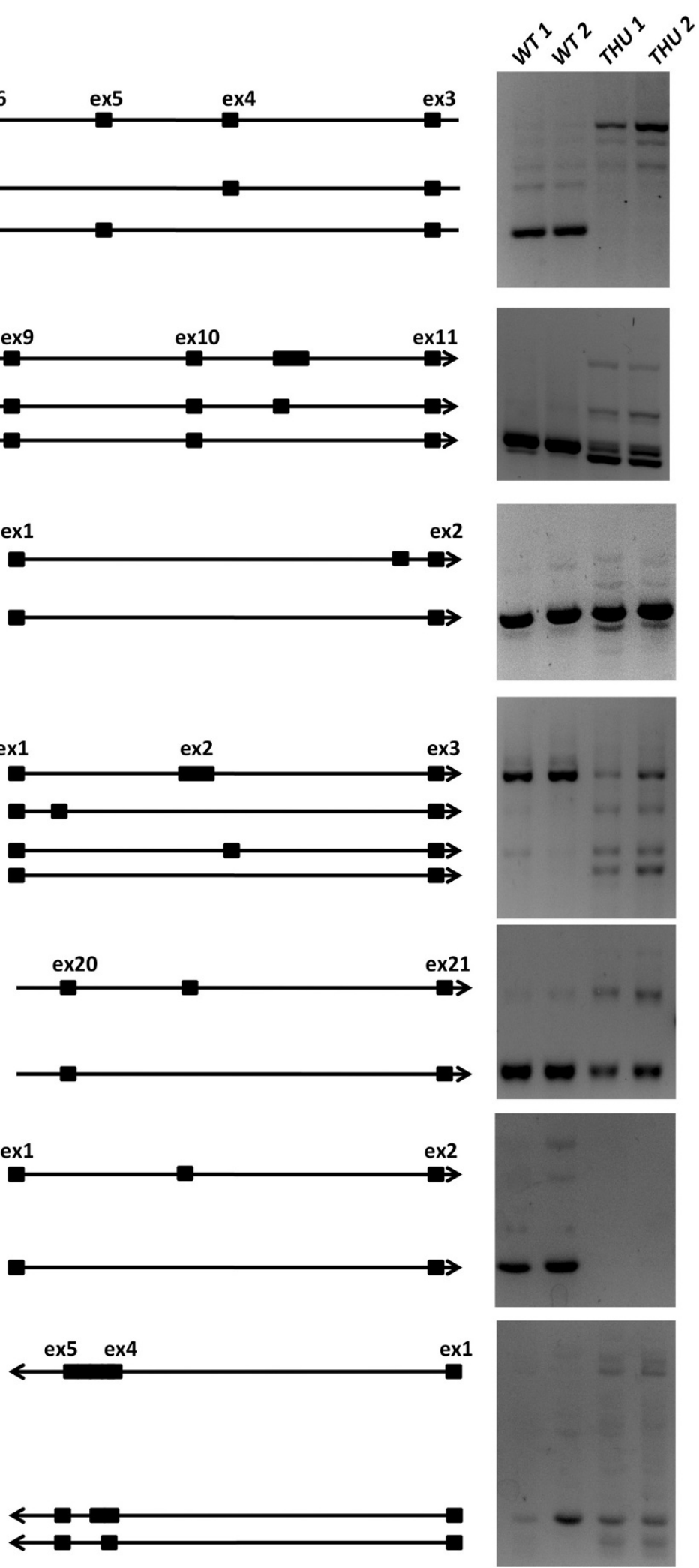

Figure 5 (See legend on next page.) 
(See figure on previous page.)

Figure 5 Altered mRNA splicing between wild-type and mutant Hnrpll CD8 ${ }^{+}$T cells validated with RT-PCR. A selection of ten genes that ranked highly in one or more tests for differential alternative splicing in Hnrplthu $T$ cells were validated using RT-PCR. Six genes showed differential PCR bands between samples and are show alongside an ideogram of the inferred sequence included in each product, determined from band sizes and the expected included sequence indicated by RNA-seq junction read information. (a) Ptprc gene, oligonucleotide PCR primers located in exons 2 and 7, amplifying across the regions of alternative exons 4, 5 and 6. (b) Senp2 gene, primers located in exons 8 and 11, amplifying the intron between exons 10 and 11 containing a variably included unannotated or cryptic exon. The sequence of each band in the accompanying ideogram was confirmed by Sanger sequencing. (c) Ctse gene, primers located in exon 1 and 2, amplifying unannotated exon in intron 1. (d) Trpv2 gene, primers located in exons 1 and 3 to amplify unannotated exons in introns 1 and 2. (e) Ash1/ gene, primers located in exons 20 and 21, spanning variably included unannotated exon in intron 20. (f) $\mathrm{S} / \mathrm{c} 12 a 7$ gene, primers located in an alternative first exon, such that preference to the other first exon there would be no product. (g) $L c k$ gene, primers were designed with a forward primer spanning across exons 1 and 4 and a reverse primer in exon 5, amplifying products of exon 1 and 4 joining (skipping exons 2 and 3) with variable length of exon 4. Oligonucleotide primer locations are displayed against read depth and gene intron/structure in Figure S2 in Additional file 4. WT, wild type.

these high ranking genes confirmed by RT-PCR. Across these genes and those identified earlier, gene set enrichment analysis did not indicate significant shared Gene Ontology terms, common pathways or other functional annotations.

\section{Normally spliced Senp2 cDNA restores Hnrplt ${ }^{\text {thu }}$ T-cell survival}

The lifespan of Hrnpll $l^{\text {thu }}$ homozygous T cells is greatly shortened, resulting in decreased numbers of naïve $\mathrm{T}$ cells in the circulation [16] due to effects on hnRNPLL targets other than Ptprc [22]. We focused on Senp2 as an additional candidate, since it was highly ranked and validated with strong evidence of intron inclusion and cryptic splicing that would decrease the pool of normal Senp2 protein in the analysis of Hrnplt ${ }^{\text {thu }}$ homozygous OT- 1 T cells above, and because it is an essential regulator of protein sumoylation with the capacity to affect many aspects of cell survival in non-lymphoid cells [31,32]. OT-1 CD8 T cells with wild-type or homozygous mutant Hnrpll were activated briefly in culture, transduced with a retroviral vector encoding normally spliced Senp2 cDNA or an empty vector control, and reimplanted into normal C57BL/6 mice. Individual transferred CD8 T cells were enumerated in blood samples collected from the recipients on day 3 and again on day 17, using flow cytometric staining for a donor-specific CD45.1 marker (Figure 7a). Wild-type T cells transduced with Senp2 or empty vector were frequent in the circulation on day 3, and persisted at $40 \%$ of this frequency on day 17. Hnrpll mutant $\mathrm{T}$ cells transduced with empty vector were less frequent on day 3 , and had declined to $11 \%$ of this frequency by day 17 , consistent with previous evidence that these $\mathrm{T}$ cells have a very short in vivo lifespan [16]. By contrast, mutant $\mathrm{T}$ cells transduced with Senp 2 vector were present at higher frequency on day 3 and persisted at $45 \%$ of this frequency by day 17 . Improved survival of Hnrpll mutant T cells transduced with Senp2 compared with control vector, but not of Hnrpll wild-type $\mathrm{T}$ cells transduced with the same vector combination, was observed in two independent experiments
(Figure 7b). Flow cytometric staining of the persisting Hnrpll mutant $\mathrm{T}$ cells showed that they nevertheless continued to be unable to silence Ptprc exon 6, displaying 10 times higher CD45RC on their cell surface compared with the transferred wild-type T-cell controls (Figure 7c). This result, together with isolation of an ENU-induced exon 10-11 splicing mutation in Senp2 that also results in shortened T-cell survival (Yan Mei, Anselm Enders, Edward M Bertram and Christopher C Goodnow, unpublished observations), provide evidence that Senp2 is an important functional target for Hnrplldependent T-cell survival.

\section{Discussion}

Our study develops a strategy for identifying regulated mRNA splicing events with RNA-seq of total polyA + RNA, using one of the best defined mammalian models of developmentally regulated alternative splicing in the Ptprc gene and a mutation that cripples hnRNPLL, a key RBP regulating this event. We have shown that introns flanking the excluded exons in this gene are retained in oligo-dT primed cDNA from $\mathrm{T}$ cells that express hnRNPLL, whereas these introns are efficiently spliced out of most of the mRNA in lymphocytes with defective or naturally low expression of hnRNPLL. Intron retention promoted by hnRNPLL appears to be a general feature of the transcriptome in T cells and we identified and validated a further 14 genes showing a similar pattern of intron retention and alternative splicing. Functional evidence for the importance of one of the highest-ranked additional targets, Senp2, was obtained by retroviral cDNA expression restoring survival of Hnrpll-mutant T cells. The computational methodology we have applied to identify hnRNPLLdependent splicing events in comparison between wild type and mutant cells is readily generalizable to identify the program of other regulatory RBPs that dictate patterns of alternative splicing, using small samples of total RNA that are readily obtained from purified ex vivo subsets of primary mammalian cell types.

We have shown that partially spliced polyadenylated mRNAs, rather than being artifacts of RNA-seq data, 
Table 2 All candidate hnRNPLL-regulated splicing events prioritized for RT-PCR confirmation

\begin{tabular}{|c|c|c|c|c|c|c|c|c|c|}
\hline \multirow[t]{2}{*}{ Gene } & \multirow[t]{2}{*}{ Intron location } & \multicolumn{6}{|c|}{ Feature scores } & \multirow[t]{2}{*}{ Score } & \multirow[t]{2}{*}{ Ranl } \\
\hline & & 3'SS ratio & 5'SS ratio & $\begin{array}{c}\text { Differential } \\
\text { exon }\end{array}$ & $\begin{array}{c}\text { Differential } \\
\text { intron }\end{array}$ & $\begin{array}{c}\text { Differential } \\
\text { junction }\end{array}$ & $\begin{array}{c}\text { Cryptic } \\
\text { junctions }\end{array}$ & & \\
\hline Ptprc & 1:140013603-140014330 & 1 & 1 & 1 & 1 & 1 & 1 & 6 & 1 \\
\hline Degs1 & 1:184207025-184208627 & 1 & 1 & 1 & 1 & 1 & 1 & 6 & 2 \\
\hline Ptprc & 1:140016744-140019032 & 1 & 1 & 1 & 1 & 1 & 0 & 5 & 3 \\
\hline Ptprc & 1:140012220-140013530 & 1 & 0 & 1 & 1 & 1 & 1 & 5 & 4 \\
\hline Ptpre & 1:140019162-140022879 & 1 & 0 & 1 & 1 & 1 & 0 & 4 & 6 \\
\hline SIc12a7 & 13:73870786-73901144 & 1 & 0 & 0 & 1 & 1 & 1 & 4 & 7 \\
\hline SIc12a7 & 13:73901339-73921966 & 0 & 1 & 0 & 1 & 1 & 1 & 4 & 8 \\
\hline Ptprc & 1:140014472-140016596 & 1 & 1 & 1 & 1 & 1 & 0 & 5 & 11 \\
\hline Rab3gap2 & 1:187087739-187090779 & 1 & 0 & 0 & 1 & 1 & 1 & 4 & 13 \\
\hline $117 r$ & 15:9440015-9440865 & 0 & 0 & 1 & 1 & 0 & 1 & 3 & 15 \\
\hline Ctse & 1:133535135-133558452 & 0 & 0 & 1 & 1 & 0 & 1 & 3 & 16 \\
\hline Sidt1 & 16:44258155-44259545 & 0 & 0 & 0 & 1 & 1 & 1 & 3 & 17 \\
\hline $117 r$ & 15:9438538-9439920 & 0 & 0 & 1 & 1 & 0 & 1 & 3 & 20 \\
\hline $117 r$ & 15:9438114-9438461 & 0 & 0 & 1 & 1 & 0 & 1 & 3 & 21 \\
\hline Ash11 & 3:88871164-88872908 & 0 & 1 & 0 & 1 & 0 & 1 & 3 & 23 \\
\hline Senp2 & 16:22032238-22036542 & 1 & 1 & 0 & 1 & 0 & 1 & 4 & 24 \\
\hline Rnf167 & 11:70463837-70464265 & 0 & 1 & 0 & 0 & 1 & 1 & 3 & 26 \\
\hline D14Abble & 14:28263236-28272964 & 0 & 0 & 0 & 0 & 1 & 1 & 2 & 33 \\
\hline Sept7 & 9:25104073-25105396 & 0 & 0 & 0 & 0 & 1 & 1 & 2 & 37 \\
\hline Mapkapk3 & 9:107166096-107182976 & 0 & 1 & 0 & 1 & 0 & 1 & 3 & 44 \\
\hline Trim68 & 7:109828211-109828650 & 1 & 1 & 0 & 0 & 1 & 1 & 4 & 48 \\
\hline Sigirr & $7: 148281217-148286377$ & 1 & 0 & 0 & 0 & 1 & 1 & 3 & 50 \\
\hline Trpv2 & 11:62388103-62389048 & 1 & 0 & 0 & 0 & 1 & 1 & 3 & 62 \\
\hline Herc3 & 6:58824395-58826482 & 1 & 0 & 0 & 1 & 0 & 1 & 3 & 66 \\
\hline $\operatorname{lghg} 2 \mathrm{C}$ & 12:114524254-114525607 & 0 & 1 & 1 & 1 & 0 & 0 & 3 & 71 \\
\hline Pik3r4 & $9: 105580651-105584514$ & 0 & 0 & 0 & 1 & 0 & 1 & 2 & 80 \\
\hline Ikbke & 1:133162866-133166377 & 0 & 0 & 0 & 1 & 0 & 1 & 2 & 83 \\
\hline Cep110 & 2:35013309-35015767 & 0 & 0 & 0 & 0 & 1 & 1 & 2 & 84 \\
\hline Fcrl1 & 3:87185307-87185682 & 0 & 0 & 0 & 0 & 1 & 1 & 2 & 87 \\
\hline Mllt6 & 11:97538551-97539627 & 0 & 0 & 0 & 1 & 0 & 1 & 2 & 104 \\
\hline Raplgds1 & 3:138619284-138620373 & 0 & 0 & 0 & 0 & 1 & 1 & 2 & 131 \\
\hline Lck & 4:129234700-129234869 & 0 & 0 & 0 & 0 & 1 & 1 & 2 & 163 \\
\hline
\end{tabular}

Number of alternative splicing event types mapped within 1) all exons in expressed genes and 2) exons having at least $10 \%$ of intron retention in their 3 'SS, and the mean number of the event types within randomly sampled exon sets of the same size.

serve as markers of alternatively spliced exons and that the retention of introns in these transcripts is a regulated process. Previous studies of nascent mRNA have employed technically demanding nuclear fractionation before RNA isolation $[7,8,33]$. Our results are built on an emerging literature that demonstrates incompletely spliced, polyadenylated mRNAs - including unspliced introns 3 to 7 in Ptprc mRNA from macrophages - are frequently retained on chromatin as well as being present in the nucleoplasm $[8,10,33,34]$. Incompletely spliced, polyadenylated mRNAs are readily identified in RNAseq datasets generated from whole-cell mRNA preparations $[10,35,36]$ and differential intron retention between different tissues and cell types has also been observed $[7,13,36]$. The cause of the differing intron excision in these studies is mostly unresolved and may stem from various factors, including intron length, strength of $5^{\prime}$ splice sites, RNA polymerase II pausing, and the binding of SR proteins to introns [33]. Our study addresses this question by establishing a strong correlation between 


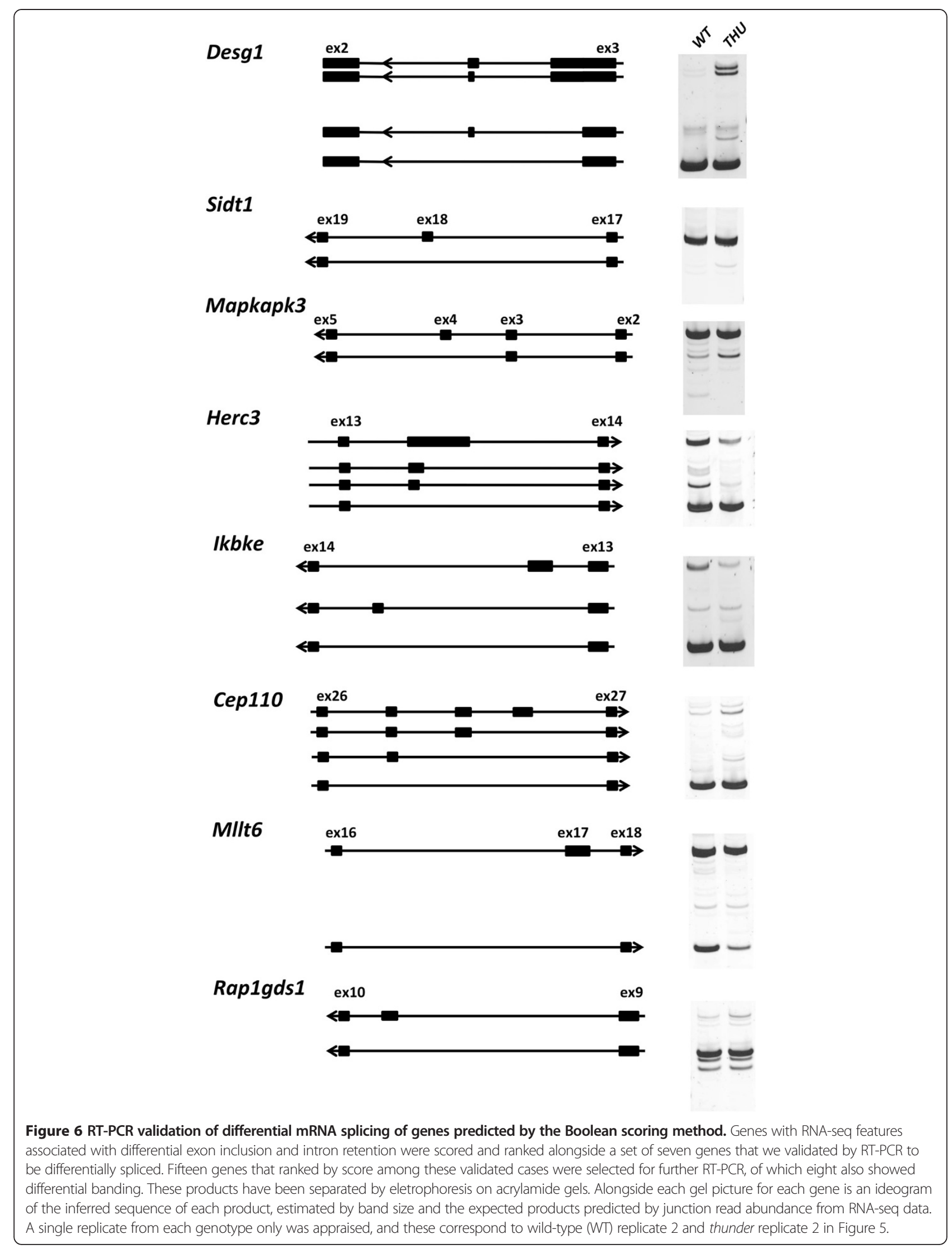



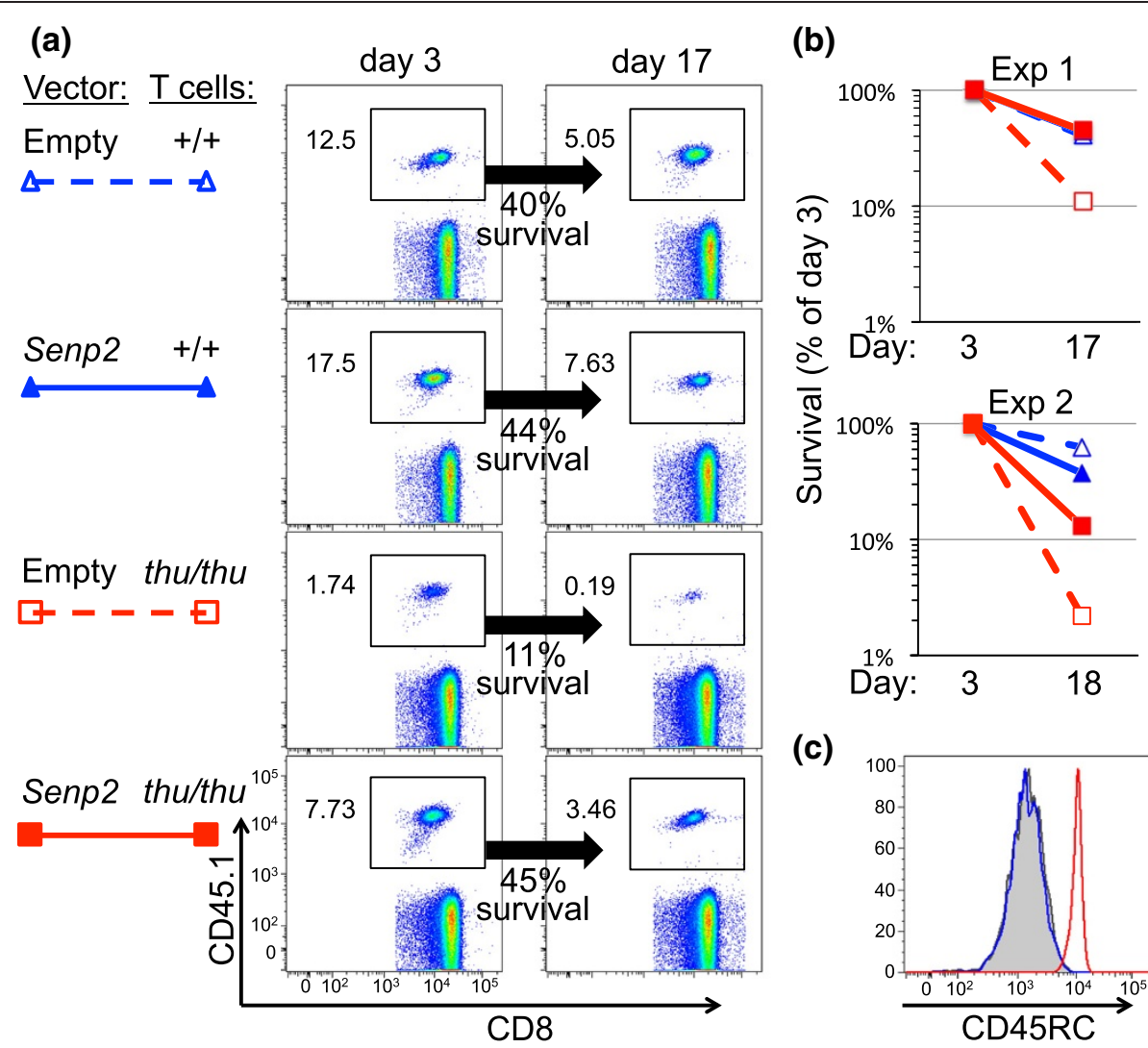

(c)

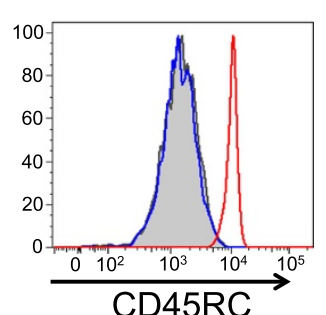

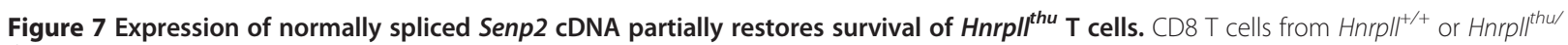
thu OT-1 TCR-transgenic B6.SJL-CD45.1/CD45.2 mice were activated and spinoculated with defective retroviral particles containing either empty retroviral vector or the same vector bearing full length, normally spliced Senp2 CDNA, and injected into the circulation of normal C57BL/6 mice. (a) Blood from each recipient mouse was analyzed by flow cytometry 3 and 17 days later, enumerating the frequency of donor CD45.1+ T cells among CD8 cells at each timepoint and the percentage that survived between days 3 and 17. (b) Data from two independent experiments analyzed as in (a). (c) Staining of the donor-derived T cells for CD45RC: black and blue histograms, +/+ cells with empty or Senp2 vectors, respectively; red histogram, thu/thu cells with Senp2 vector.

intron retention in total polyA + RNA and the presence of variably spliced exons, and by experimentally demonstrating the action of a developmentally regulated, RNAbinding splicing silencer, hnRNPLL. Nuclear fractionation and sequencing of nascent, chromatin-associated transcripts and those from the nucleoplasm will provide a higher degree of sensitivity for detecting unspliced transcripts, but the high quantity of input material required is unfeasible for cells of limited abundance. The diversity of RBPs in mammalian cells allows strong cell-type-specific regulation of splicing, as we have found, and produces an imperative to compare purified cell populations, which will frequently mean that starting cells will be in limited supply.

Along with this simple means of generating data we have developed a scoring and ranking scheme to identify genes displaying properties associated with regulated intron retention and alternative splicing. No single feature of exon inclusion, intron retention or splice junction usage was solely sufficient to reliably identify a group of transcripts as undergoing regulated late splicing, but a simple integration of evidence from RNA-seq data could better rank genes. From a set of six genes with RNA-seq and RT-PCR evidence for differential splicing between wild-type and thunder Hnrpll mRNAs, we identified that cryptic junction reads are a frequent feature of introns flanking alternative exons. Machine learning approaches are often used to take information about a training set of genes to extrapolate further similar cases across a large dataset. We applied a naïve Bayesian classifier to use these six genes to identify further targets of hnRNPLL regulation. Perhaps due to the small number of training genes we found the classifier did not produce a list of genes with convincing evidence on visual inspection of the RNA-seq data in IGV. As more studies elucidate the features of alternatively spliced genes regulated by RBPs, a greater number of known, training genes may enable better use of machine learning algorithms.

Regulated retention of introns flanking alternative exons in the Ptprc gene indicates that splicing decisions are frequently delayed until after transcription and 
polyadenylation. One suggested role for post-transcriptional splicing is that it provides more (or later) opportunities for regulated alternative splicing [10]. The retained introns we have observed in the Ptprc gene do not appear to be 'dead-end' transcripts destined for degradation, but are likely exons on which a splicing decision is pending. Speculatively, delayed processing of variable exons in Ptprc may provide a pool of mRNA that can be rapidly modified in response to changing extracellular signals. This paradigm is perhaps best demonstrated by the unconventional cytoplasmic splicing of $H a c 1 / X b p 1$ mRNA in response to endoplasmic reticulum stress [37], but has also been suggested for conventional alternative splicing [13]. While there is no direct evidence to support this possibility for Ptprc, TCR-induced dephosphorylation of PSF1 promotes Ptprc exon silencing [38]. Potentially, this pool of 'delayed transcripts' are awaiting a cellular decision on the splicing of alternative exons. In the absence of this decision introns surrounding the alternative exons remain unspliced, to allow the option of a splicing event that causes an exon to be skipped.

The differentially spliced genes between wild-type and thunder $\mathrm{T}$ cells elucidate the primary or secondary targets of hnRNPLL and identify a cellular program that is likely to be specifically regulated between naïve and activated/memory $\mathrm{T}$ cells. Both SR proteins and hnRNPs are encoded by large gene families and the diversity of these proteins allows highly tissue-specific splicing regulation by single RBPs [39]. Hence, we need not expect a large number of hnRNPLL-dependent targets. However, apart from these genes all being co-expressed in T cells, there are few annotations that obviously link them functionally as a larger program. Several pairwise functional associations are clear, such as the well characterized direct interaction of CD45 with LCK to dephosphorylate pTyr505 and pTyr-394 regulatory tyrosines on LCK [40,41]. One hypothesis to explain this apparent lack of a broader program may be that the true functional targets of hnRNPLL are indeed a very small set, perhaps being just one, two or three genes. Functional associations between these genes will not be effectively captured through gene set enrichment analysis, as gene pairs or trios will be statistically very sensitive to missing or incomplete functional annotations. The differential exon usage in the Ptprc gene is the clearest signal seen in the RNA-seq data and the signal of the other genes is much less distinct, and decreases quickly to the limit of detection through our positive gene list. It could be that genes with weaker signals represent minor or promiscuous binding targets of hnRNPLL without strong functional effect. Of the stronger targets, namely those in our first list of six high ranking gene targets, it will be important to investigate the functional impact of the splicing regulation mediated by hnRNPLL. We provide evidence here that inefficient splicing of one of these targets, Senp2, contributes to the short lifespan of hnRNPLL-mutant T cells. Senp2 encodes a sumo-specific protease and results in embryonic lethality when knocked out [31,32], but how it promotes T-cell longevity will await further study.

Detection of genes with differentially retained introns from RNA-seq data suggests a method for mapping the targets and extended regulatory program of hnRNPs and potentially some SR proteins. This approach identifies both direct and secondary targets of RNA binding proteins, and hence would be highly complementary to additional sources of information from RIP-seq, PAR-CLIP and other immunoprecipitation followed by sequencing approaches. Collections of mice with knockout and mutant hnRNPs are steadily growing and transcriptome data taken from appropriate cells could be extracted, sequenced and mined using a technically simple approach similar to that presented here. For example, hnRNPLL and hnRNPL have overlapping roles in regulating the splicing of Ptprc in T cells [42]. With existing mouse knockouts for hnRNPL, mapping of the functional targets of RBPs could be undertaken similarly to this study, in combination with genome-wide RNA-binding assays.

\section{Conclusions}

Collectively, our data indicate that lymphocytes that express wild-type hnRNPLL at high levels (wild-type T cells) exhibit a delay in splicing of the Ptprc introns surrounding those exons (exons 4 and 6) with multiple hnRNPLL binding sequences, so that 8 to $20 \%$ of mRNA retains these introns after they have spliced out most other introns and become polyadenylated. This provides in vivo genetic evidence in mammalian cells that intron processing is delayed around alternatively spliced exons in nascent mRNA, whereas it often occurs co-transcriptionally and preceding polyadenylation for introns associated with constitutively spliced exons, consistent with emerging observations in other systems $[7,8,33]$.

\section{Materials and methods RNA preparation}

Single cell suspensions of mouse spleens were prepared by sieving and gentle pipetting followed by lysis of red blood cells. CD8 ${ }^{+} \mathrm{T}$ cells were isolated to $>90 \%$ purity (as assessed by flow cytometry) using mouse CD8 $\alpha$ microbeads (Miltenyi Biotec Australia Pty. Ltd. NSW, Australia) following the manufacturer's instructions. Total RNA was then extracted using TRIzol (Invitrogen ${ }^{\mathrm{Tm}}$ Life Technologies Australia Pty. Ltd. Victoria, Australia) and isopropanol precipitation. The purity and integrity of RNA was determined using the Agilent Bioanalyzer, which reported an RNA integrity number of $>8.5$ for all samples. $\mathrm{CD} 19^{+} \mathrm{B}$ cells and $\mathrm{CD} 4^{+} \mathrm{T}$ cells were purified from spleens of wild-type C57BL6 mice using 
microbeads conjugated with either anti-CD19 or antiCD4 antibodies.

\section{Sequencing and mapping}

Sequencing was done using an Illumina GAIIx with a single-end length of $125 \mathrm{bp}$ for OT1 samples and $76 \mathrm{bp}$ for non-OT1 samples. RNA-sequencing reads were aligned to the mouse reference genome $\mathrm{mm} 9$ (NCBIM37) using TopHat (v1.4.1) with Bowtie by using default parameters, which allows up to two mismatches [23]. Mean base coverage of an intron or exon was calculated as following: Number of reads mapped $\times$ Read length/Feature length. Gene expression was estimated from all reads mapping to all exons annotated to a gene. A minimum average coverage threshold of 20 bases was used to designate genes as expressed. The raw sequencing data have been uploaded to NCBI Short Read Archive under accession number SRP034881.

\section{Detection of differential splicing}

Identification of differential inclusion of introns, exons and/or splice junctions was done using the DEXSeq package (version 1.8.0 on $R$ version 3.0.2) [28], which normalizes count data for different coverage depths and estimates data dispersions, then tests for differential intron/exon/junction usage between wild type and mutant using a generalized linear model. An input dataset of nonoverlapping exonic parts of the $\mathrm{mm} 9$ genome was created by the script dexseq_prepare_annotation.py provided in the package based on the Ensembl transcript annotation file Mus_musculus.NCBIM37.66.gtf, which was used to create a table of intronic parts for multi-exon genes. For more sensitive detection of differential reads within long introns, introns were further divided into $300 \mathrm{bp}$ windows. The dexseq_count.py script, also part of the DEXSeq package, was then used to count the number of reads that fall within each exon or intron. Analysis of differential exon and intron inclusion were performed on both features simultaneously with DEXSeq (Additional file 5). As most introns contain few or no reads and pooling the data in this manner allowed better normalization. Dispersion estimation and statistical model calculation was performed using the TRT method provided with DEXSeq on exons and introns with a minimum of 10 counts over all samples. A raw splicing junction count dataset was generated using TopHat and was used in DEXSeq analysis to find differential splice junction usage for junctions of a minimum count of 2 over all samples. At a false discovery rate of $0.1,558$ out of 230,416 junctions were found to be significant.

\section{Calculation of intron retention ratio}

Intron retention ratios across $5^{\prime}$ and $3^{\prime}$ splice sites were calculated as the sum of reads mapped to $25 \mathrm{bp}$ upstream and downstream of the splice site, and calculated as the ratio of intronic reads to exonic reads, as previously described [7].

\section{Retained introns and annotated alternative splicing events}

Exons associated with high 3 ' - or $5^{\prime}$ 'SS read ratios were compared to alternative splicing events annotated to the Ensembl mouse gene set [26] (NCBIM37, Ensembl release 67). Annotated alternative splicing event types included constitutive exon (CNE), cassette exon (CE), mutually exclusive exons (MXE), intron retention (IR), alternative 3' sites (A3SS), and alternative $5^{\prime}$ sites (A5SS) after the nomenclature described in [43]. A frequency of each splicing event type was calculated for exons that had a 3'SS ratio $\geq 0.01$ in wild-type T cells from OT1 mice, then compared with 1,000 randomly permuted sets for each type of splicing event. $P$-values were calculated for a null hypothesis that the frequencies of splicing event types in exons having at least $10 \%$ of intron retention are not different to a background distribution, with an alternative hypothesis that the frequencies in exons of increased 3'SS IR is higher for event types CE, MXE, IR, A3SS, and A5SS and lower for the CNE type.

\section{Scoring scheme to rank differential intron retention events}

Six measures related to differential intron retention were calculated: differential exon inclusion, differential splice junction usage, differential intron retention, distinct cryptic splice sites (per intron) and 3' - and 5'SS ratios. Differential exon, intron and splice junction read tests were calculated with DEXSeq as described above and features were scored 'true' if they were below a significance threshold equivalent to a false discovery rate of less than 0.1. Cryptic junctions were scored as 'true' if they had more than one unannotated junction supported by three or more reads in at least one sample. $3^{\prime}$ - and $5^{\prime}$ SS ratios were calculated as described above and these ratios compared between cells types. Where ratios differed by 0.1 or more, these were scored as 'true'. Across all six feature types true values were summed and introns ranked according to total score.

\section{PCR validation of candidate genes}

RNA samples from OT-1 $\mathrm{CD}^{+} \mathrm{T}$ lymphocytes from spleen of wild-type and thunder mice were prepared as described above then reverse transcribed to produce first-strand cDNAs (Marligen Biosciences, Ijamsville, MD, USA). cDNA products were quantified with a Nanodrop Spectrophotometer and used for PCR amplification of candidate genes using Taq DNA polymerase with a $55^{\circ} \mathrm{C}$ annealing temperature and 35 cycles, which were then electrophoresed in $2 \%$ agarose gel or $4 \%$ 
polyacrylamide gel, stained with GelRed or Syber Gold, respectively. Primer sequences used for validation of alternative splicing variants are shown in Table S4 in Additional file 4.

\section{OT-1 T cell transduction and transplantation}

CD8 $\mathrm{T}$ cells were purified by magnetic bead depletion of other lymphocyte subsets from spleens of OT-1 TCR transgenic B6.SJL-CD45.1 congenic mice of $\mathrm{Hnrpll}^{+/+}$or Hnrpll ${ }^{\text {thu/thu }}$ genotype. The T cells were activated by culture at $2 \times 10^{6}$ cells $/ \mathrm{ml}$ with plate-bound anti-CD3 and soluble anti-CD28 antibodies for $24 \mathrm{~h}$, and then spinoculated with defective retroviral particles packaged in Phoenix cells containing either empty pMIG II vector or the same vector bearing full length normally spliced Senp 2 cDNA. The T cells were placed back in culture with anti-CD3 and anti-CD28 for $24 \mathrm{~h}$, washed, and injected into the lateral tail vein of normal C57BL/6 mice. Each recipient mouse was bled 3 and 17 days later, blood cells stained for CD8, CD45.1 and CD45RC, and analyzed by flow cytometry.

\section{Additional files}

Additional file 1: Table S1. Table of differential intron usage found by DEXSeq.

Additional file 2: Table S2. Table of differential exon usage found by DEXSeq.

Additional file 3: Table S3. Table of differential junction usage found by DEXSeq.

Additional file 4: Figure S1. A Venn diagram of genes with features (exon/intron/junction) found significant by DEXSeq. DEXSeq tests for finding differential exon, intron and junction inclusion resulted in a total of 127, 102, and 401 genes with significant features, respectively. Genes identified by all three tests are listed in group $\mathrm{A}$, and by two tests are in group B, C and D. Figure S2. Representation of coverage depth and splice junction usage in genes with validated alternative splicing events by PCR. Figure S3. A cryptic exon inclusion in Hnrp/lthunder $T$ cells is confirmed by sequencing PCR bands of Senp2. Table S4. A list of primer sequences used in validation of candidate genes with alternative splicing variants.

Additional file 5: $\mathrm{R}$ log files for DEXSeq analysis of finding differential intron/exon and junction usage.

\section{Abbreviations}

ARS: activation response sequence; bp: base pair; hnRNP: heterogeneous ribonucleoprotein; IGV: Integrative Genomics Viewer; IR: intron retention; RBP: RNA-binding protein; RRM: RNA-recognition motif; RT-PCR: reverse transcriptase polymerase chain reaction; SR protein: Serine/arginine-rich protein; SS: splice site; TCR: T-cell receptor.

\section{Competing interests}

The authors declare that they have no competing interests.

\section{Authors' contributions}

VC, AT, CCG and TDA designed the study. VC, YM, AT and AS performed the experiments. AM, SC, AE and EB produced and maintained mouse breeding lines. VC and TDA analyzed the data. VC, CCG and TDA wrote the paper. All authors read and approved the final manuscript.

\section{Authors' information}

Christopher C Goodnow and T Daniel Andrews are senior authorship.

\section{Acknowledgements}

This work has been supported by grants from the National Health and Medical Research Council (Australia), the Wellcome Trust, the National Institutes of Health (USA) and the Biomedical Research Council (BMRC) of the Agency for Science, Technology and Research (A*STAR), Singapore.

\section{Author details}

${ }^{1}$ Immunogenomics Laboratory, John Curtin School of Medical Research, Australian National University, GPO Box 334, Canberra City ACT 2601, Australia. ${ }^{2}$ Immunology Groups, Bioprocessing Technology Institute, 20 Biopolis Way, Centros, 138668 Singapore, Singapore. ${ }^{3}$ Microarray Groups, Bioprocessing Technology Institute, 20 Biopolis Way, Centros, 138668 Singapore, Singapore. ${ }^{4}$ Ramaciotti Immunisation Genomics Laboratory, John Curtin School of Medical Research, Australian National University, GPO Box 334, Canberra City ACT 2601, Australia. ${ }^{5}$ Australian Phenomics Facility, Hugh Ennor Building, Australian National University, Garran Road, ACT 0200 Canberra, Australia.

Received: 17 October 2013 Accepted: 29 January 2014

Published: 29 January 2014

\section{References}

1. Wahl MC, Will $C L$, Lührmann R: The spliceosome: design principles of a dynamic RNP machine. Cell 2009, 136:701-718.

2. Black DL: Mechanisms of alternative pre-messenger RNA splicing. Annu Rev Biochem 2003, 72:291-336.

3. Matlin AJ, Clark F, Smith CWJ: Understanding alternative splicing: towards a cellular code. Nat Rev Mol Cell Biol 2005, 6:386-398.

4. Martinez NM, Lynch KW: Control of alternative splicing in immune responses: many regulators, many predictions, much still to learn. Immunol Rev 2013, 253:216-236.

5. Moore MJ, Proudfoot NJ: Pre-mRNA processing reaches back to transcription and ahead to translation. Cell 2009, 136:688-700.

6. Oesterreich FC, Bieberstein N, Neugebauer KM: Pause locally, splice globally. Trends Cell Biol 2011, 21:328-335.

7. Khodor YL, Rodriguez J, Abruzzi KC, Tang C-HA, Marr MT, Rosbash M Nascent-seq indicates widespread cotranscriptional pre-mRNA splicing in Drosophila. Genes Dev 2011, 25:2502-2512.

8. Bhatt DM, Pandya-Jones A, Tong A-J, Barozzi I, Lissner MM, Natoli G, Black DL, Smale ST: Transcript dynamics of proinflammatory genes revealed by sequence analysis of subcellular RNA fractions. Cell 2012, 150:279-290.

9. Khodor YL, Menet JS, Tolan M, Rosbash M: Cotranscriptional splicing efficiency differs dramatically between Drosophila and mouse. RNA 2012, 18:2174-2186.

10. Tilgner H, Knowles DG, Johnson R, Davis CA, Chakrabortty S, Djebali S, Curado J, Snyder M, Gingeras TR, Guigó R: Deep sequencing of subcellular RNA fractions shows splicing to be predominantly co-transcriptional in the human genome but inefficient for IncRNAs. Genome Res 2012, 22:1616-1625.

11. Das R, Dufu K, Romney B, Feldt M, Elenko M, Reed R: Functional coupling of RNAP II transcription to spliceosome assembly. Genes Dev 2006, 20:1100-1109.

12. de la Mata M, Lafaille C, Kornblihtt AR: First come, first served revisited: factors affecting the same alternative splicing event have different effects on the relative rates of intron removal. RNA 2010, 16:904-912.

13. Pandya-Jones A, Black DL: Co-transcriptional splicing of constitutive and alternative exons. RNA 2009, 15:1896-1908.

14. Hermiston $M L, X u Z$, Weiss $A: C D 45$ : a critical regulator of signaling thresholds in immune cells. Annu Rev Immunol 2003, 21:107-137.

15. Oberdoerffer S, Moita LF, Neems D, Freitas RP, Hacohen N, Rao A: Regulation of CD45 alternative splicing by heterogeneous ribonucleoprotein, hnRNPLL. Science 2008, 321:686-691.

16. Wu Z, Jia X, de la Cruz L, Su X-C, Marzolf B, Troisch P, Zak D, Hamilton A, Whittle B, Yu D, Sheahan D, Bertram E, Aderem A, Otting G, Goodnow CC, Hoyne GF: Memory T cell RNA rearrangement programmed by heterogeneous nuclear ribonucleoprotein hnRNPLL. Immunity 2008, 29:863-875 
17. Topp JD, Jackson J, Melton AA, Lynch KW: A cell-based screen for splicing regulators identifies hnRNP LL as a distinct signal-induced repressor of CD45 variable exon 4. RNA 2008, 14:2038-2049.

18. Rothrock C, Cannon B, Hahm B, Lynch KW: A conserved signal-responsive sequence mediates activation-induced alternative splicing of CD45. Mol Cell 2003, 12:1317-1324.

19. Rothrock CR, House $A E$, Lynch $K W$ : HnRNP L represses exon splicing via a regulated exonic splicing silencer. EMBO J 2005, 24:2792-2802.

20. Tong A, Nguyen J, Lynch KW: Differential expression of CD45 isoforms is controlled by the combined activity of basal and inducible splicingregulatory elements in each of the variable exons. J Biol Chem 2005, 280:38297-38304

21. Gaudreau M-C, Heyd F, Bastien R, Wilhelm B, Möröy T: Alternative splicing controlled by heterogeneous nuclear ribonucleoprotein $L$ regulates development, proliferation, and migration of thymic pre-T cells. $\mathrm{J}$ Immunol 2012, 188:5377-5388.

22. Wu Z, Yates AL, Hoyne GF, Goodnow CC: Consequences of increased CD45RA and RC isoforms for TCR signaling and peripheral T cell deficiency resulting from heterogeneous nuclear ribonucleoprotein L-like mutation. J Immunol 2010, 185:231-238.

23. Trapnell C, Pachter L, Salzberg SL: TopHat: discovering splice junctions with RNA-Seq. Bioinformatics 2009, 25:1105-1111.

24. Heng TSP, Painter MW, Consortium IGP: The immunological genome project: networks of gene expression in immune cells. Nat Immunol 2008, 9:1091-1094.

25. ImmGen microarray gene expression data: Data Generation and Quality Control pipeline. [http://www.immgen.org/Protocols/ImmGen\%20QC\% 20Documentation_ALL-DataGeneration_0612.pdf]

26. Flicek P, Ahmed I, Amode MR, Barrell D, Beal K, Brent S, Carvalho-Silva D, Clapham P, Coates G, Fairley S, Fitzgerald S, Gil L, García-Girón C, Gordon L, Hourlier T, Hunt S, Juettemann T, Kähäri AK, Keenan S, Komorowska M, Kulesha E, Longden I, Maurel T, McLaren WM, Muffato M, Nag R, Overduin B, Pignatelli M, Pritchard B, Pritchard E, et al: Ensembl 2013. Nucleic Acids Res 2013, 41:D48-D55.

27. Katz $Y$, Wang ET, Airoldi EM, Burge CB: Analysis and design of RNA sequencing experiments for identifying isoform regulation. Nat Methods 2010, 7:1009-1015

28. Anders $S$, Reyes A, Huber W: Detecting differential usage of exons from RNA-seq data. Genome Res 2012, 22:2008-2017.

29. Thorvaldsdóttir H, Robinson JT, Mesirov JP: Integrative genomics viewer (IGV): high-performance genomics data visualization and exploration. Brief Bioinformatics 2013, 14:178-192.

30. Benson DA, Karsch, ÄêMizrachi I, Lipman DJ, Ostell J, Wheeler DL: GenBank: update. Nucleic Acids Res 2004, 32:D23-D26.

31. Chiu SY, Asai N, Costantini F, Hsu W: SUMO-specific protease 2 is essential for modulating p53-Mdm2 in development of trophoblast stem cell niches and lineages. PLOS Biol 2008, 6:e310.

32. Kang X, Qi Y, Zuo Y, Wang Q, Zou Y, Schwartz RJ, Cheng J, Yeh ET: SUMO-specific protease 2 is essential for suppression of polycomb group protein-mediated gene silencing during embryonic development. Mol Cell 2010, 38:191-201.

33. Pandya-Jones A, Bhatt DM, Lin CH, Tong AJ, Smale ST, Black DL: Splicing kinetics and transcript release from the chromatin compartment limit the rate of Lipid A-induced gene expression. RNA 2013, 19:811-827.

34. Brody $Y$, Neufeld N, Bieberstein N, Causse SZ, Böhnlein E-M, Neugebauer KM, Darzacq X, Shav-Tal Y: The in vivo kinetics of RNA polymerase II elongation during co-transcriptional splicing. PLOS Biol 2011, 9:e1000573.

35. Ameur A, Zaghlool A, Halvardson J, Wetterbom A, Gyllensten U, Cavelier L, Feuk $L$ : Total RNA sequencing reveals nascent transcription and widespread co-transcriptional splicing in the human brain. Nat Struct Mol Biol 2011, 18:1435-1440.

36. Wong JJ, Ritchie W, Ebner OA, Selbach M, Wong JW, Huang Y, Gao D, Pinello N, Gonzalez M, Baidya K, Thoeng A, Khoo TL, Bailey CG, Holst J, Rasko JE: Orchestrated intron retention regulates normal granulocyte differentiation. Cell 2013, 154:583-595.

37. Sidrauski C, Cox JS, Walter P: tRNA ligase is required for regulated mRNA splicing in the unfolded protein response. Cell 1996, 87:405-413.

38. Heyd F, Lynch KW: Phosphorylation-dependent regulation of PSF by GSK3 controls CD45 alternative splicing. Mol Cell 2010, 40:126-137.

39. Busch A, Hertel KJ: Evolution of SR protein and hnRNP splicing regulatory factors. Wiley Interdiscip Rev RNA 2012, 3:1-12.
40. D'Oro U, Sakaguchi K, Appella E, Ashwell JD: Mutational analysis of Lck in CD45-negative T cells: dominant role of tyrosine 394 phosphorylation in kinase activity. Mol Cell Biol 1996, 16:4996-5003.

41. Veillette A, Caron L, Fournel M, Pawson T: Regulation of the enzymatic function of the lymphocyte-specific tyrosine protein kinase p56lck by the non-catalytic SH2 and SH3 domains. Oncogene 1992, 7:971-980.

42. Preussner $M$, Schreiner $S$, Hung L-H, Porstner M, Jäck H-M, Benes V, Rätsch G, Bindereif A: HnRNP L and L-like cooperate in multiple-exon regulation of CD45 alternative splicing. Nucleic Acids Res 2012, 40:5666-5678.

43. Wang ET, Sandberg R, Luo S, Khrebtukova I, Zhang L, Mayr C, Kingsmore SF, Schroth GP, Burge CB: Alternative isoform regulation in human tissue transcriptomes. Nature 2008, 456:470-476.

\section{doi:10.1186/gb-2014-15-1-r26}

Cite this article as: Cho et al:: The RNA-binding protein hnRNPLL

induces a $T$ cell alternative splicing program delineated by differential intron retention in polyadenylated RNA. Genome Biology 2014 15:R26.

\section{Submit your next manuscript to BioMed Central and take full advantage of:}

- Convenient online submission

- Thorough peer review

- No space constraints or color figure charges

- Immediate publication on acceptance

- Inclusion in PubMed, CAS, Scopus and Google Scholar

- Research which is freely available for redistribution

Submit your manuscript at www.biomedcentral.com/submit
C) Biomed Central 